\title{
Review
}

\section{MRI and M/EEG studies of the White Matter Development in Human Fetuses and Infants: Review and Opinion}

\author{
Jessica Dubois $^{\mathrm{a}, *}$, Parvaneh Adibpour ${ }^{\mathrm{a}}$, Cyril Poupon ${ }^{\mathrm{b}}$, Lucie Hertz-Pannier ${ }^{\mathrm{c}}$ \\ and Ghislaine Dehaene-Lambertz ${ }^{\mathrm{a}}$ \\ a INSERM, UMR992; CEA, NeuroSpin Center; University Paris Saclay, Gif-sur-Yvette, France \\ ${ }^{\mathrm{b}}$ CEA, NeuroSpin Center, UNIRS; University Paris Saclay, Gif-sur-Yvette, France \\ ${ }^{\mathrm{c} C E A}$, NeuroSpin Center, UNIACT; University Paris Saclay, Gif-sur-Yvette, France; INSERM, \\ UMR1129; University Paris Descartes, Paris, France
}

\begin{abstract}
Already during the last trimester of gestation, functional responses are recorded in foetuses and preterm newborns, attesting an already complex cerebral architecture. Then throughout childhood, anatomical connections are further refined but at different rates and over asynchronous periods across functional networks. Concurrently, infants gradually achieve new psychomotor and cognitive skills. Only the recent use of non-invasive techniques such as magnetic resonance imaging (MRI) and magneto- and electroencephalography (M/EEG) has opened the possibility to understand the relationships between brain maturation and skills development in vivo. In this review, we describe how these techniques have been applied to study the white matter maturation. At the structural level, the early architecture and myelination of bundles have been assessed with diffusion and relaxometry MRI, recently integrated in multi-compartment models and multi-parametric approaches. Nevertheless, technical limitations prevent us to map major developmental mechanisms such as fibers growth and pruning, and the progressive maturation at the bundle scale in case of mixing trajectories. At the functional level, M/EEG have been used to record different visual, somatosensory and auditory evoked responses. Because the conduction velocity of neural impulses increases with the myelination of connections, major changes in the components latency are observed throughout development. But so far, only a few studies have related structural and functional markers of white matter myelination. Such multi-modal approaches will be a major challenge in future research, not only to understand normal development, but also to characterize early mechanisms of pathologies and the influence of fetal and perinatal interventions on later outcome.
\end{abstract}

Keywords: Brain organization, architecture, maturation, myelination, bundles, diffusion tensor imaging DTI, relaxometry, evoked potentials or fields, sensory modalities

\footnotetext{
${ }^{*}$ Correspondence to: Jessica Dubois, $\mathrm{PhD}, \mathrm{CEA} / \mathrm{SAC} / \mathrm{DRF} /$ I2BM/NeuroSpin/Cognitive Neuroimaging Unit U992, Bât 145, point courrier 156, 91191 Gif-sur-Yvette, France. Tel.: +33 1 690894 98; Fax: +33 1 690879 73; E-mail: jessica. dubois@centraliens.net.
}

\section{ABBREVIATIONS}

$\begin{array}{ll}\text { AEPs } & \begin{array}{l}\text { auditory evoked potentials } \\ \text { blood-oxygen level dependant } \\ \text { BOLD }\end{array} \\ \text { CHARMED } & \begin{array}{l}\text { composite hindered and restricted } \\ \text { model of diffusion }\end{array}\end{array}$




$\begin{array}{ll}\text { CST } & \text { cortico-spinal tract } \\ \text { DKI } & \text { diffusion kurtosis imaging } \\ \text { DTI } & \text { diffusion tensor imaging } \\ \text { DWI } & \text { diffusion-weighted imaging } \\ \text { EEG } & \text { electroencephalography } \\ \text { FA } & \text { fractional anisotropy } \\ \text { GW } & \text { gestational weeks (GW = weeks of } \\ & \text { amenorrhea } \approx \text { PCW }+2) \\ \text { HARDI } & \text { high angular resolution diffusion } \\ & \text { imaging } \\ \text { MEG } & \text { magnetoencephalography } \\ \text { MRI } & \text { magnetic resonance imaging } \\ \text { MTI } & \text { magnetization transfer imaging } \\ \text { MTR } & \text { magnetization transfer ratio } \\ \text { MWF } & \text { myelin water fraction } \\ \text { NODDI } & \text { neurite orientation dispersion } \\ & \text { and density imaging } \\ \text { PCW } & \text { post-conception weeks } \\ \text { PMW } & \text { post-menstrual weeks (PMW }= \\ & \text { gestational age at birth }+ \\ & \text { post-natal weeks) } \\ \text { R1 } & \text { relaxation rate associated with T1 } \\ & \text { relaxation time (R1 = 1/T1) } \\ \text { R2 } & \text { relaxation rate associated with T2 } \\ & \text { relaxation time (R2 = 1/T2) } \\ \text { SEFs } & \text { somatosensory evoked fields } \\ \text { SEPs } & \text { somatosensory evoked potentials } \\ \text { S1 } & \text { primary somatosensory cortex } \\ \text { T1 } & \text { longitudinal relaxation time } \\ \text { T1w } & \text { T1-weighted } \\ \text { T2 } & \text { transverse relaxation time } \\ \text { T2w } & \text { T2-weighted } \\ \text { VEFs } & \text { visual evoked fields } \\ \text { VEPs } & \text { visual evoked potentials } \\ \lambda / / & \text { longitudinal diffusivity } \\ \lambda \perp & \text { transverse diffusivity } \\ & \end{array}$

\section{INTRODUCTION}

The development of functional networks in the human brain starts during the fetal life, and the early functional architecture rapidly shares similarities with descriptions at older ages. For examples, studies of spontaneous blood-oxygen level dependant (BOLD) activity with functional MRI have identified most resting-state networks during the preterm period, with relatively comparable properties than later on $[1,2]$. Already at 6 months of gestation, listening to auditory syllables elicit organized responses in peri-sylvian regions as in the adult brain [3, 4]. These early functional responses attest that the macro- and microstructural development is already sufficient during the last trimester of gestation to allow the efficient transfer and processing of information across brain regions.

All major long-distance fibers, which are formed sequentially from the 9th post-conception week (PCW), are observed by the end of the late preterm period. After term birth, these anatomical connections are further refined through several complementary mechanisms. Useless and redundant axonal fibers are pruned by the end of the first post-natal year, whereas the ongoing myelination process stabilizes functionally relevant connections and increases the efficiency of the information transfer between distant brain regions. The number of oligodendrocytes and astrocytes drastically increases in the white matter during the first 3 years of life, attaining two-thirds of the corresponding numbers in adults, whereas the number of neurons and microglia remains almost the same [5]. The inhibitory role of oligodendrocytes and myelin on neuritic growth may partly explain the weak plasticity of the adult brain [6]. In addition to the early brain organization in specific networks, a major developmental characteristic is the asynchronous progression of maturation across brain areas: for instance sensory regions develop early on and quickly, whereas associative regions are slowly developing until the end of adolescence.

Concurrently with this anatomical evolution of the brain, the infant gradually achieves new psychomotor and cognitive skills. Nevertheless, the complex links between brain maturation and new behavioural skills are still poorly understood. Before the booming of brain imaging techniques, our knowledge on human brain development relied on post-mortem investigations, which are intrinsically limited by the lack of anatomo-functional correlations. Animal studies remain largely inadequate because of the specificity of human cognitive development. Only the recent use of non-invasive techniques such as magnetic resonance imaging (MRI), magneto- and electroencephalography (M/EEG) or near-infrared spectroscopy (NIRS) has opened the possibility to relate the maturation of cerebral networks and infants' behaviour. But applying these techniques to babies is challenging and requires adapting both data acquisition and post-processing procedures to deal with short examinations, motion issues, and signal changes throughout maturation.

This article aims to outline how the development of white matter bundles, notably the progression of myelination, has been studied in vivo with MRI 
and M/EEG. As detailed extensively in our previous review articles [7,8], a plethora of studies has addressed this topic in the recent years. Rather than systematically reviewing them again, we here focus on the most recent findings in order to specifically outline challenges and pitfalls of each approach. We successively detail how structural MRI studies have enabled to map the early organization (I) and the asynchronous maturation (II) of white matter bundles at the macroscopic level. The reader may refer to [7] for additional details on the specificities of fetal and infant imaging and for basic explanations on the evolution of MRI parameters throughout development. We then describe how M/EEG studies have assessed the functional development of these connections, and how structural and functional markers of maturation, provided by MRI and M/EEG respectively, can be tightly related (III). All along this review, we discuss conceptual and technical issues that remain to be solved to integrate the complexity of brain networks development. We hope that our questioning will trigger potential glimpses for future directions of research.

\section{THE EARLY ORGANIZATION OF WHITE MATTER PATHWAYS}

The knowledge on bundles growth has classically relied on post-mortem histological examinations. Recent advances in MRI now enable to map this process both in ex utero specimens and in vivo thanks to diffusion imaging approaches such as diffusion tensor imaging (DTI), high angular resolution diffusion imaging (HARDI) combined with sophisticated tractography algorithms to infer the direction of fibers in 3D.

\section{Post-mortem identification of the developing bundles}

Because this section is mainly a prerequisite to understand the following in vivo findings (I.2.), we here summarize the developmental descriptions that have been reported on post-mortem specimen so far, whatever the investigation technique: histological examinations, anatomical and diffusion MRI (see [7-10] for further details). These approaches provide complementary information, each one being intrinsically limited to identify the tracts precisely (particularly MRI as detailed in I.3.). Consequently this global report strongly relies on the current state of technological art.
Before the formation of stable postnatal connectivity, transient fetal patterns of connectivity are observed [8-10]. During the early fetal period (9$15 \mathrm{PCW}$ ), major fiber pathways are growing and pathfinding within the intermediate zone. Some afferent (e.g. thalamo-cortical fibers in the internal capsule) and efferent (cortico-subcortical) fibers are already observed. Before penetrating into the cortical plate, thalamo-cortical fibers make connections with neurons of the subplate zone, a crucial structure that develops between 13 and $15 \mathrm{PCW}$ [11]. Several limbic bundles (e.g. fornix, cingulum) are also identified [9], and their entire trajectories are visible at $17 \mathrm{PCW}$ with diffusion imaging and tractography [12].

During the midfetal period (15-23PCW), major efferent fibers penetrate their targets in the striatum, the pons and the spinal cord. Periventricular crossroads are developing, notably with the growth of cortico-cortical callosal and associative fibers within the intermediate zone [13]. The corpus callosum, the uncinate and inferior longitudinal fasciculi are detected with DTI [14]. The radial coherence of white matter observed at these ages with HARDI likely reflects a mixture of radial glial fibers, penetrating blood vessels, and radial axons [15]. In specimens younger than $20 \mathrm{PCW}$, the radial migration pathway seems more developed posteriorly than anteriorly [16]. Recently, axonal tracts were also identified with digitalization and structure tensor analysis of histological sections stained with antibody against neural cell adhesion molecule (NCAM) [17]. At 20PCW, the intermediate zone contains different populations of fibers: bundles of fibers with a predominant tangential orientation, arching in a C-shape from the exit of the internal capsule around and parallel to the lateral ventricle toward the corpus callosum, and a more diffusely organized fraction of fibers with radial orientation [17].

During the early preterm period (24-28PCW), afferent fibers that were waiting in the subplate start making connections within the cortical plate in most brain regions, leading to the establishment of the permanent connectivity with neurons of the future cortical layer IV. Nevertheless a transient fetal circuitry still exists within the subplate [18], and this compartment reaches its maximum volume and thickness around 30PCW [19]. Limbic cortico-cortical connections are well developed in the cingulate, entorhinal and hippocampal cortices [20]. In the lateral neocortex, the fronto-occipital and uncinate fascicles are visible [21, 22]. 
During the late preterm period (29-34 PCW), long associative and commissural bundles are quickly developing, likely originating from pyramidal neurons of cortical layer III [23]. Short cortico-cortical fibers are growing and entering the cortex through the remnant subplate [21, 24].

After term birth, U-fibers develop [22], and cortico-cortical connectivity is reorganized by several processes including the development of dendritic arborization, the overproduction of synapses and dendritic spines and their later elimination [25-27]. Pruning of axonal fibers like callosal connections [28] also probably extends until the end of the first postnatal year [11, 22, 29].

\section{In vivo identification of bundles with diffusion MRI}

In vivo diffusion MRI of fetal and preterm brains has confirmed these post-mortem studies but with lower spatial resolution. In utero, the pyramidal tract, the splenium and genu of the corpus callosum are observed early on from 18 gestational weeks (GW) [30-32]. The uncinate and inferior fronto-occipital fascicles are depicted as early as $20 \mathrm{GW}$, while the inferior longitudinal fasciculus, cingulum and fornix can be visualized in 3D in older fetuses during the third trimester [33]. In preterms, subcortical projection and association bundles are also identified [34, 35].

In the recent years, the network architecture of the preterm brain has also been detailed based on connectivity matrices, by measuring for all pairs of brain regions, the degree of anatomical connections (i.e. the number of reconstructed streamlines, eventually weighted by DTI anisotropy as a local measure of the connections strength and integrity). Already at 30 post-menstrual weeks (PMW), such a structural connectome demonstrates a small-world modular organization like in the adult brain [36]. Cortical hubs are highly connected to form a "rich club" that may underlie early network communication [37]. Until term-equivalent age, the small-world topology further increases [36], and the network structure gets more clustered between 27 and 45PMW, with the apparent development of many connections [38]. While these approaches of structural connectivity can provide whole-brain descriptions of networks without any a priori anatomical assumption on specific pathways, their biological interpretation throughout development should remain cautious relatively to plausible neural connectivity and myelination (see
[39] for a more general discussion on the anatomical reality of small-world architecture reported in brain imaging studies).

During early infancy, although weakly myelinated, all major white matter bundles are identified with diffusion imaging and tractography: commissural bundles of the corpus callosum (genu, body and splenium), projection bundles (cortico-spinal and spino-thalamic tracts, optic radiations, anterior limb of the internal capsule), limbic bundles (fornix and cingulum) and associative bundles (external capsule, uncinate, arcuate, superior and inferior longitudinal fascicles) [40, 41] (Figs. 1, 2, 4a-d). The trajectory and morphology of these bundles remain stable, as revealed by a longitudinal study, between birth and 2 years of age [42]. Short-range connections, such as the ones projecting above and below the superior temporal sulcus, are also depicted in the infant brain by 3T-MR imaging at high spatial resolution [43] (Fig. 4c). At the whole brain level, an increase in global efficiency and integration, and a decrease in segregation are observed on the structural connectome during the first two post-natal years [44].

\section{Caveats}

Several issues should be kept in mind when diffusion imaging and tractography are used to decipher the developing organization of white matter fibers. First, estimating the real axonal trajectories is not so simple, and notably during the fetal life to disentangle them from the radial glia fibers. The trajectory of reconstructed fibers is highly dependent on the acquisition parameters (e.g. the number of directions), the diffusion model and the tractography approach, and results may not be comparable across acquisition protocols, across studies, or even across ages due to the growing brain size, the myelination and density of axons, and the complex crossings of bundles with other structures (e.g. other bundles, glial fibers). DTI and deterministic tractography can highlight relatively simple trajectories, but only more elaborated models (e.g. HARDI) and algorithms (e.g. deterministic with regularization, probabilistic) can highlight accurate and realistic tracts (see Fig. 2a,b showing reconstructions of the cortico-spinal tract). Furthermore, a bundle may not be dissected, particularly in the developing brain, because of an insufficient spatial resolution compared to its small size, or because of its low maturation compared with surrounding fibers. When bundles are crossing, the situation is even worse, and exact trajectories may hardly be 


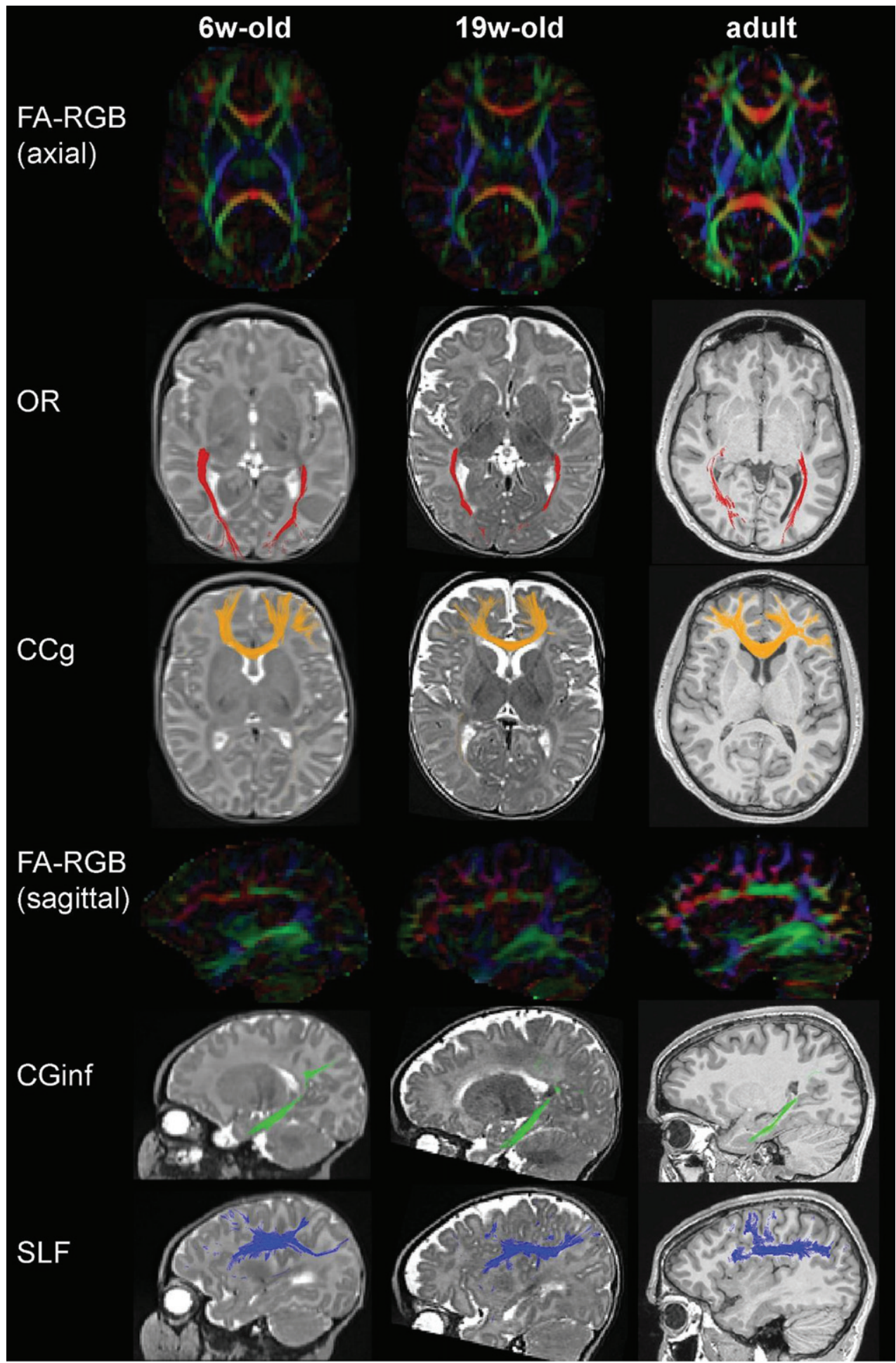

Fig. 1. Main bundles assessed by tractography are similar in early life and adulthood. DTI color-coded directionality maps (FA-RGB) for two infants of different ages ( 6 and 19 weeks old) and a young adult, on axial and sagittal views, and tract reconstructions superposed to anatomical images (T2-weighted in infants, T1-weighted in adult) for four examples of bundles (projection: optic radiations OR, commissural: genu of the corpus callosum CCg, limbic: inferior branch of the cingulum CGinf, association: superior longitudinal fasciculus SLF). Other examples of tracts can be found in [7, 40, 43]. 


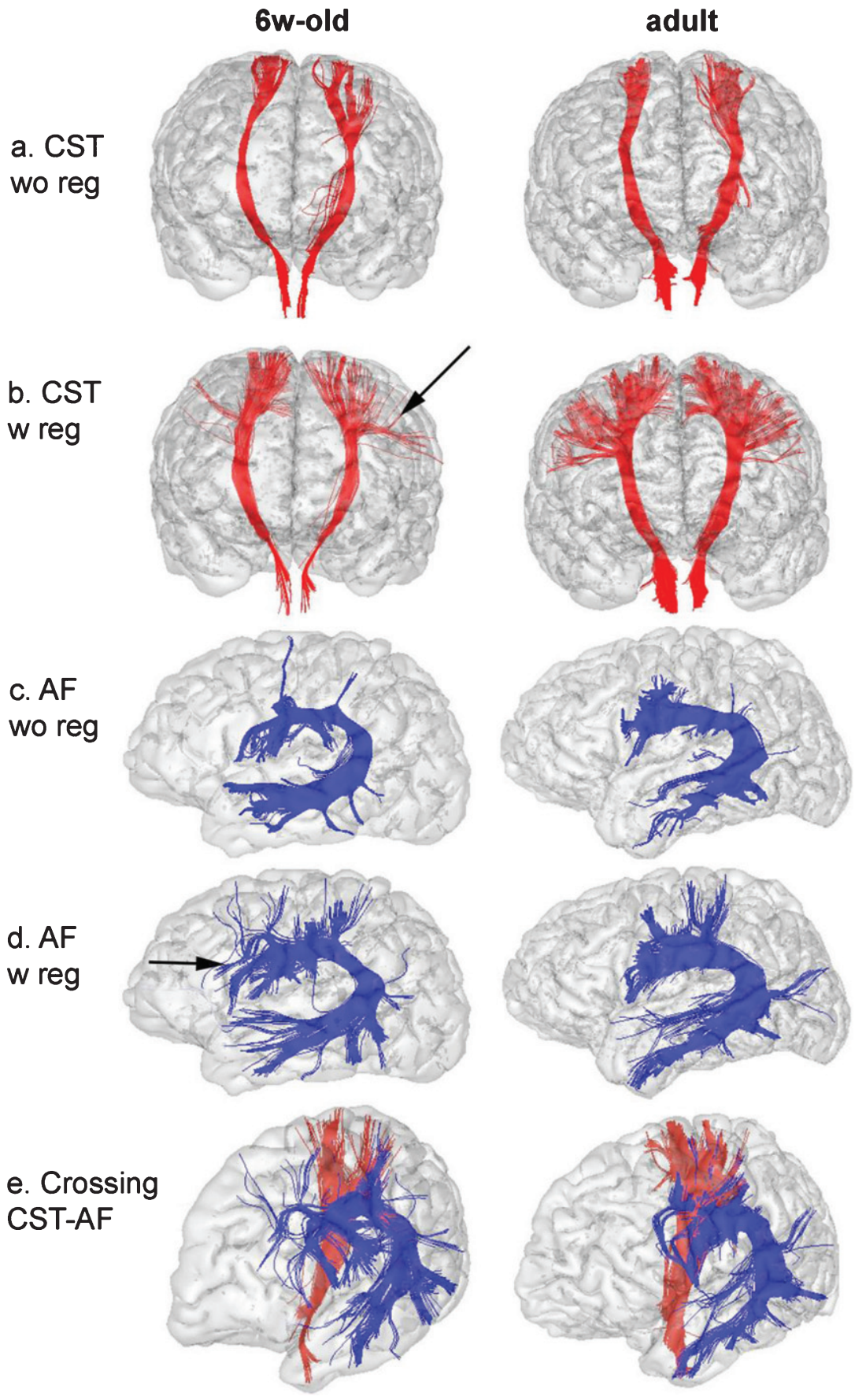

Fig. 2. Influence of tractography algorithms on the tracts reconstruction. a,b: Reconstructions of the cortico-spinal tracts (CST) on coronal view in a 6-week-old infant and a young adult, obtained by deterministic tractography (a) and by an algorithm with regularization in low anisotropy voxels [151] (b). With such an approach, the fanning of fibers in the corona radiata is better depicted along the homunculus of the central sulcus. In infants, it is mostly the case for projections at the level of cortical regions controlling the mouth and tongue (arrow), perhaps because of advanced maturation. c,d: Reconstructions of the arcuate fasciculus (AF) with the same tractography algorithms presented on sagittal view. Except for some frontal terminations (arrow), relatively similar trajectories are obtained across algorithms, maybe because the fibers orientation are more coherent than for cortico-spinal fibers. e: Superposition of the cortico-spinal tract and arcuate fasciculus, showing the crossings at the level of the corona radiata. Note that these particular reconstructions are not very different between the infant and adult, but that a high variability was observed across the infants group in the arcuate reconstructions (data not shown). 
delineated. Besides, diffusion imaging is not sensitive to the functional direction of fibers that is from the neural soma to the periphery or from region A to $B$. Thus, to which direction the fiber is growing cannot be directly inferred at the early stages of brain development. One can only try to assess whether connections exist or not between regions $\mathrm{A}$ and $\mathrm{B}$.

Second, there is no simple way so far to measure fibers overproduction and pruning, yet a major developmental process. Throughout infancy, macroscopic changes in bundles volume are mostly driven by the increase in axonal diameter and fiber myelination, rather than by fiber loss. In the infrequent event that fibers are identified at an early stage but not at a more mature stage, one may wonder whether it reflects a genuine pruning phenomenon or artefactual differences in tract reconstructions (see Figs. 1 and 2 for comparative reconstructions in infants and adults). Similarly as for the tract trajectories, the number of reconstructed streamlines strongly depends on acquisition and post-processing parameters. It is impacted by effective spatial resolution relatively to brain size, by white matter maturation as detailed below, etc. Thus this measure should be considered with caution notably in connectivity matrices, and it may provide little information on the real axonal count.

Third, the bundle maturational stage highly affects the tracts delineation. On the one hand, the tight organization of fibers in compact and highly coherent bundles leads to intrinsic anisotropy even in the absence of myelin [45], for instance in the corpus callosum of preterm newborns [46]. Studies in rat pups have shown that the first evidence of anisotropy precedes initial myelin [47], and that this early anisotropy may be related to sodium-channel activity [48]. But on the other hand, myelination highly increases the fibers anisotropy (see part II), and thus the contrast between the bundle, surrounding tissue and crossing fibers. This is particularly an issue when one aims to assess whether connections exist or not in the developing brain. It should be kept in mind that reconstructed fibers definitely remain a rough representation of axonal fibers. Recently, Li and colleagues [49] described with DTI an increase in the density of fibers connecting the cortex (projecting to or originating from it) during the first post-natal year. Rather than a recognized biological phenomenon, their observation probably relies on the maturation of fibers that already exist at birth but could not be tracked. In the same way, weakly mature bundles may appear shorter on tractography reconstructions in infants than in adults, just because of the low anisotropy at the distant extremity related to the myelination progression from the cell soma to the axonal end. This phenomenon may lead to erroneous interpretation on the presence/absence of a pathway. For instance, the arcuate fasciculus may wrongly seem not to fully connect the posterior temporal and inferior frontal regions in newborns [50, 51], as a result from an artefact due to the low myelination of its frontal extremity [43] (Fig. 2c,d). The reconstruction becomes even more complex at places where bundles maturing over different time-periods do cross. It is for example the case for the crossing of the arcuate fasciculus and cortico-spinal tract at the level of the corona radiata (Fig. 2e). The same phenomena may explain why some late-maturing associative bundles (e.g. the superior longitudinal fasciculus) display changes in fiber orientations during the first post-natal months [52] though they have grown prenatally. Finally, these caveats (e.g. missing or shorter connections) also highly impact connectome approaches. In regards to these different issues, section II.5 summarizes the bottlenecks to be solved in order to accurately track bundles of the immature brain despite fiber pruning, bundles crossing and asynchronous maturation.

\section{THE MYELINATION OF WHITE MATTER PATHWAYS}

\section{Current knowledge based on histological examinations}

Concurrently and subsequently to the organization of general neural connectivity, the white matter bundles progressively mature and become functionally more efficient through myelination (i.e. myelin formation around axons). This process includes several steps, and proceeds from the neuron body to the periphery [53]. It occurs in the human brain from the second part of pregnancy to the end of adolescence, with a peak during the first post-natal year, and it develops at different ages and rates depending on the bundles and the networks [54-56]. Thus the myelination progression varies across cerebral regions [56-62]: it follows a caudo-rostral gradient, progresses from the center to the periphery, and it occurs earlier and faster in sensory pathways (somatosensory, vision, audition) than in motor ones, and in projection fibers than in associative ones. Leftright asynchronies are also observed [43, 63], which are interesting to consider in relation to the functional 
asymmetries of the motor, linguistic and social cognition networks.

To classify white matter regions according to their myelination patterns, eight sub-groups have been proposed. Based on post-mortem examinations, they integrate whether myelin is present (A) or not (B) at birth, and the age periods at which mature myelin is observed (stages 1-4) [57]. For instance, the corticospinal tract in the midbrain and pons, the posterior limb of the internal capsule and the central corona radiata belong to sub-group A1; optic and auditory radiations, the corpus callosum body and splenium belong to sub-group B1; the anterior limb of the internal capsule, the external capsule and cingulum belong to sub-group B2, etc.

\section{In vivo quantification based on MRI parameters}

Since a few years, several MRI parameters (Fig. 3) have been used to quantify the myelination process in the developing white matter [7]. As detailed below, intense changes are measured after term-birth and during the first post-natal months (Fig. 3 right row), then the rate of modifications slows down during toddlerhood and more drastically thereafter until young adulthood.

With myelination, water content in voxels decreases, thus the proton density decreases, while the macromolecule tissue volume [64] increases [65]. This mechanism, together with changes in water molecules compartmentalization [66] and increase of protein and lipid contents [67,68], lead to decreases in $\mathrm{T} 1$ and $\mathrm{T} 2$ relaxation times with age [69-71] (Fig. 3a,b), with different time courses between T1 and T2 [72]. Indeed, T1 shortening starts first during the "pre-myelinating" state, while T2 shortening occurs later on with the chemical maturation of the myelin sheath [56, 67, 73]. Myelinated white matter regions may already be found on $\mathrm{T} 1 \mathrm{w}$ and $\mathrm{T} 2 \mathrm{w}$ images during the preterm period, mainly from $36 \mathrm{w}$ GA on [74], but changes are mainly seen after term and vary across the brain because of the asynchronous myelination process $[40,65]$.

DTI parameters capture some aspects of this maturational pattern $[75,76]$. During the preterm period, diffusivities decrease, while anisotropy increases in most white matter regions [77] except at cross-roads locations [78]. During infancy and childhood, transverse diffusivity decreases more than longitudinal diffusivity [42, 79, 80] (Fig. 3d,e), and anisotropy keeps increasing (Fig. 3c) except where the crossing bundles are at different maturational stages creating an ambiguous signal [7]. These parameters might be sensitive to distinct microstructural mechanisms such as the proliferation of glial cell bodies and extension of oligodendroglial processes during the first stage of myelination ("pre-myelination") [81, 82 ], and the ensheathment of oligodendroglial processes around the axons during the "true" myelination process [79]. Among DTI parameters, transverse diffusivity is probably the best marker of myelination [83-85]. Aside from the myelination effects, anisotropy and longitudinal diffusivity are more dependent on the tract intrinsic architecture (e.g. fibers compactness, crossings) than transverse diffusivity. Indeed, the variability in anisotropy and longitudinal diffusivity observed across bundles seem relatively stable during infancy and adulthood, despite important age-related changes [7, 43, 86]. Like for T1 and T2, DTI parameters demonstrate an asynchronous evolution across bundles [79], even within a functional network [43] (Fig. 4c). A centralto-peripheral and posterior-to-anterior gradient of maturation is observed during the preterm period [77], as well as a posterior-to-anterior gradient during childhood [80].

The progression of myelination along tracts can be exquisitely studied with tractography-based quantification of DTI parameters, as recently reported for main bundles during toddlerhood [87], childhood and adolescence [88]. For instance, during the preterm period, the cortico-spinal tract starts maturing at the level of the internal capsule [89]. During infancy, the optic radiations show an early central-to-peripheral wave of maturation, followed by a peripheral-to-central wave, which might correspond to thalamo-cortical and cortico-thalamic fibers respectively [86]. Because DTI parameters are also affected by the tract shape, compactness, and by the crossings and partial volume effects with neighboring structures, such analyses require a normalization of the values by the same measures done at the mature stage to disentangle maturation per se from these other factors $[7,43,79,86]$.

Several other MRI parameters can also be measured to quantify the myelin amount in white matter. Magnetic susceptibility decreases during childhood with maturation [90]. Magnetization transfer imaging (MTI) is sensitive to hydrogen atoms bound to macromolecules, such as proteins and lipids found in myelin [91], and magnetization transfer ratio (MTR) increases with myelination [68], showing asynchrony across white matter regions [92]. However, dur- 

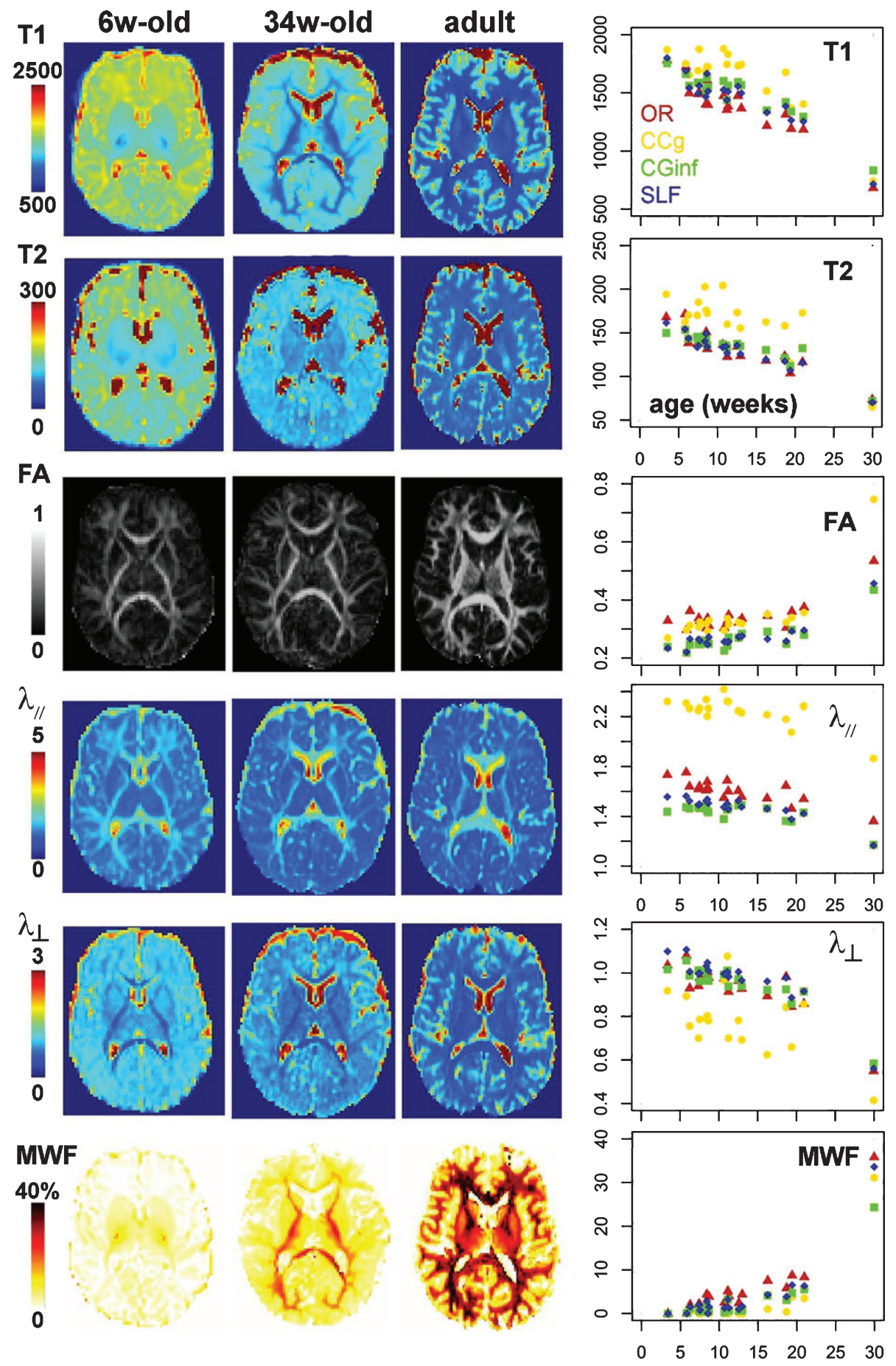

Fig. 3. Asynchrony and spatial heterogeneity of maturation quantified with MRI parameters. Left rows: Maps of MRI parameters (a: T1, b: T2, c: DTI fractional anisotropy FA, d: longitudinal diffusivity $\lambda_{/ /}$, e: transverse diffusivity $\lambda_{\perp}$, f: myelin water fraction MWF) in a 6and a 34-week-old infant, and a young adult. Right row: Age-related changes in MRI parameters over a group of 17 infants aged between 3 and 21 weeks, for the four white matter bundles shown in Fig. 1 (projection: optic radiations OR, commissural: genu of the corpus callosum $\mathrm{CCg}$, limbic: inferior branch of the cingulum CGinf, association: superior longitudinal fasciculus SLF), suggesting different maturational calendar across bundles. The median values computed over a group of 13 young adults are also indicated on the right of the plots (arbitrary age of $30 \mathrm{w})$. There is a high variability across bundles also at the mature stage particularly in terms of FA, $\lambda / /$ and MWF. For quantification on other bundles, one can refer to [40,99]. 


\section{a. Bundles maturation}

\section{Projection bundles}
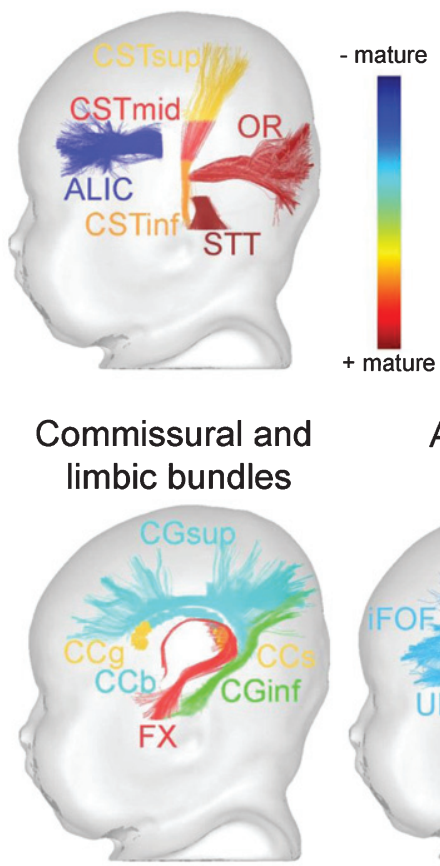

b.

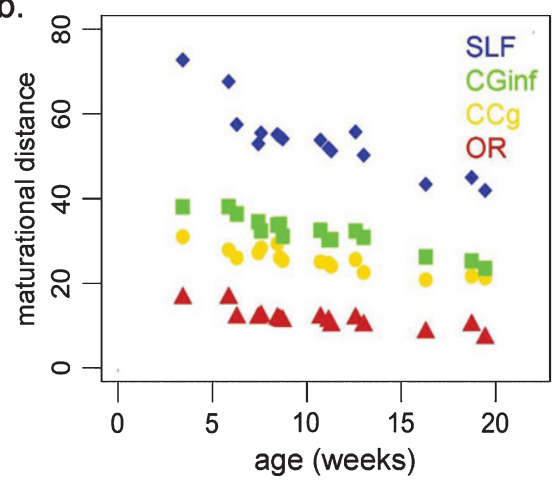

c. Within the language network
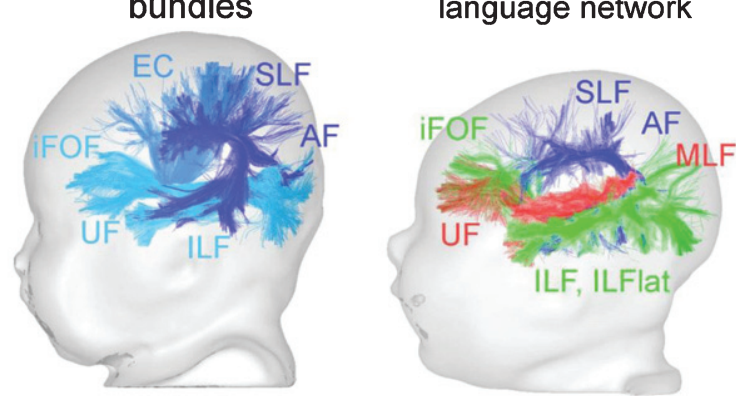

Fig. 4. Asynchronous maturation of white matter bundles based on multi-parametric assessment of MRI parameters. a: Relative maturation of projection, commissural, limbic and association bundles during infancy (from the most mature in red to the least mature in blue), evaluated from 3 to 21 weeks of age by a multi-parametric approach that computes the maturational distance relative to the adult stage (adapted from $[8,40]$ ). b. Age-related changes of this maturational distance for the four bundles presented in Figs. 1 and 3 (adapted from [40]). c. Relative maturation within the language network, showing advanced maturation of the ventral pathway (uncinate, middle and inferior longitudinal, inferior fronto-occipital fascicles) compared to the dorsal pathway (arcuate and superior longitudinal fascicles) during infancy (adapted from [43]). The color scales of maturation are not comparable in a. and c. Abbreviations: Projection bundles: ALIC anterior limb of the internal capsule; CST cortico-spinal tract (inf/mid/sup inferior/middle/superior portions); OR optic radiations; STT spino-thalamic tract; Callosal bundles: CC corpus callosum (g/b/s genu/body/splenium); Limbic bundles: CG cingulum (inf/sup inferior/superior parts); FX fornix; Association bundles: AF arcuate fasciculus; EC external capsule; iFOF inferior fronto-occipital fasciculus; ILF inferior longitudinal fasciculus; SLF superior longitudinal fasciculus; UF uncinate fasciculus.

ing the preterm period, MTR is unexpectedly high in the unmyelinated callosal fibers [81], and it decreases in the frontal intermediate zone where cross-roads develop [78]. These observations suggest that MTR is not only sensitive to myelin-associated macromolecules, but also to the macromolecular density of axonal cytoskeleton components such as microtubules and neurofilaments. A recent study in a cuprizone mouse model of demyelination has shown that among MRI parameters (T1/T2, DTI, MTI), the bound pool fraction $(f)$ from MTI is the best indicator of the myelin sheath fraction, while $\mathrm{T} 1$ relates to fraction of myelinated axons, and longitudinal diffusivity relates to the fraction of nonmyelinated cells; these two latter parameters thus characterize the tissue micro-architecture rather than myelination [93].

\section{Refined quantification based on multi-compartment models}

Recently, several modelling approaches based on relaxometry or diffusion data have been proposed to characterize distinct pools of water molecules inside each voxel. These pools can be distinguished on the basis of their different relaxometry or diffusion characteristics. Such decomposition is supposed to provide more accurate information on the tissue microstructure than considering univariate parameters at the voxel level. Nevertheless, it requires the acquisition of many more data to estimate the pools characteristics, limiting its actual use in fetuses and infants.

On the one hand, analyses based on multicomponent relaxation (MCR) assume that at least 
two or three pools of water contribute to the MR signal sensitized to $\mathrm{T} 1$ and $\mathrm{T} 2$ relaxometries [94]: water located within the myelin sheath (with relatively short $\mathrm{T} 1$ and $\mathrm{T} 2$ relaxation times), intra-axonal, intra-cellular and interstitial water (i.e. water outside of the myelin sheath, with intermediate T1 and T2), and free water (with long T1 and T2, close to values of cerebro-spinal fluid). Different methods have been used so far in the developing brain, such as multicomponent $\mathrm{T} 2$ relaxometry [95] or multi-component driven equilibrium single pulse measures [96]. The main potential of these approaches is to estimate the volume fraction of water related to myelin (often named the "myelin water fraction" MWF). This fraction drastically increases with age in the white matter (Fig. 3f), shows a spatio-temporal progression that is coherent with histological studies of myelination $[72,97]$, and follows a nonlinear growth pattern during infancy and toddlerhood [98]. To reduce the MRI acquisition time required for the estimation of MWF maps in infants, we recently proposed an approach based on the assumption that $\mathrm{T} 1$ and $\mathrm{T} 2$ characteristics of water pools remain stable throughout development and can be fixed to adult estimates, and that only the fraction changes of the different pools account for the known sequence of $\mathrm{T} 1$ and $\mathrm{T} 2$ changes during development [99]. Such an hypothesis may be a good approximation given previous description of stable myelin chemical properties during maturation [73].

On the other hand, different multi-component approaches have been proposed to analyze the MR diffusion signal acquired with multiple shells (i.e. sensitized to multiple b-values) and with multiple gradient directions. Designed for the adult brain, these complex models (e.g. CHARMED [100] or NODDI [101]) enable to characterize the tissue microstructural properties and eventually the spatial distribution of fibers within white matter voxels, by modeling distinct water pools (e.g. water with hindered diffusion in the extra-cellular space, water with restricted diffusion in the intra-cellular compartment). Recent studies have tested these approaches in the developing brain by fixing the pools characteristics (diffusivities) to adult estimates. This is a reasonable hypothesis given that intra- and extra-cellular axial diffusivities, estimated with diffusion kurtosis imaging (DKI), appear stable throughout development [102]. In newborns, the intra-neurite volume fraction from NODDI may inform on the maturation of white matter fibers, while the index of neurite orientation dispersion seems to reflect the presence of fiber crossings and fanning [103]. Thus these parameters may help distinguish between bundles with similar cellular structure but different myelination (e.g posterior vs anterior limb of the internal capsule), or reciprocally with similar maturation but different fiber microstructural organization (e.g. external capsule vs periventricular crossroads) [103]. During the first three post-natal years, intra-axonal water fraction and tortuosity of the extra-axonal space increase, with asynchronous patterns across distinct white matter regions such as the corpus callosum genu and splenium, and the posterior limb of the internal capsule [102]. Actually, during childhood and adolescence, the increase in DTI anisotropy observed in most bundles seems to relate to the increase in NODDI neurite density rather than on changes in orientation dispersion [104]. Despite these approaches look very promising to precisely characterize the tissue microstructure, diffusion imaging only provides indirect information on the myelin amount: indeed no pool of water related to myelin can be taken into account in diffusion models because of too short $\mathrm{T} 2$ characteristics.

\section{Refined quantification based on multi-parametric approaches}

Despite some correlations between specific pairs of parameters and over specific developmental periods [7], MRI parameters (T1, T2, DTI, MTR, MWF, NODDI, etc.) do capture different tissue properties, and provide complementary information on maturation mechanisms. Thus they show different age-related trajectories (for recent example over the lifespan [65]), which makes it possible to differentiate white matter structures based on their maturation patterns (for recent example during the preterm period $[78,81])$. A clever combination or integration of these parameters may thus provide a unique opportunity to infer the bundles microstructural organization and myelination, and to put in relation networks maturation and infants' learning.

In complement to myelin-sensitive and diffusion imaging techniques, a composite framework has been proposed recently to estimate the g-ratio [105], i.e. the ratio between the inner and outer diameters of the myelin sheath, which is supposed to be a fundamental characteristic of white matter fibers. The approach relies on MTI to estimate the myelin content and on NODDI to assess the intra-axonal fraction. It provides accurate estimations of g-ratios in the corpus callosum compared with histological examinations in the monkey brain, and looks promising 
to detect demyelinating lesions in adult patients with multiple sclerosis [105]. Recently, similar approaches using MWF instead of MTR, have been tested to quantify the white matter myelination in the developing brain of newborns [106], infants and children [107]. Because g-ratio depends on at least two different MRI techniques, this parameter may be even more sensitive to acquisition protocols than previous parameters, making comparisons across studies difficult. Besides, this approach only provides a rough estimate of the complex processes involved in the development of white matter microstructure and myelination. Among unrevealed aspects, g-ratio is a voxel-wise aggregate index that, like other MRI parameters, merges the properties of distinct fibers: e.g. fibers to be pruned do not myelinate but probably may have similar properties of diameter and trajectory than myelinating fibers; crossing fibers may show different g-ratios and age-related changes according to asynchronous myelination, etc.

In the last years, a few other multi-parametric approaches have shown potential to quantify the progression of white matter maturation throughout infancy. Let us remember that MRI parameters vary across both bundles and white matter regions also in the adult brain, in relation to fibers geometry, compactness, crossings, etc. Highlighting maturational effects in the developing brain thus requires either considering the developmental trajectories toward adulthood to evaluate the asymptotes of maturation, or normalizing infant measurements by adult references. Recently, modelling the evolution of proton density, T1w and T2w signals, or DTI diffusivities has provided quantitative absolute rates of change and relative time shifts across regions [108]. Combining the time trajectories of DTI parameters has also accurately highlighted their evolution across bundles [109]. Besides, clustering brain voxels over a group of infants on the basis of T1, T2 and DTI parameters, has uncovered four types of white matter regions with different compactness and maturation [110]: the spatial distribution of these clusters was anatomically relevant while the approach was free from any anatomical hypothesis. Finally, a maturational distance to the adult stage has been proposed to summarize changes in T1, T2 and DTI diffusivities during early infancy while taking into account their possible correlations [40]. In addition to outperforming univariate approaches, this approach enables the quantitative timing of maturation asynchrony (in weeks) across projection, association, limbic and callosal bundles, showing that most intense changes occur during the first post-natal year (Fig. 4a,b).

\section{Some reflections for the accurate exploration of bundles architecture and maturation}

To sum up, several MRI techniques have been recently proposed to explore the organization and maturation of white matter networks in the fetal and infant brain. Nevertheless, several concerns have been raised on the correspondence between these measures and the biological mechanisms occurring during development, mainly because these mechanisms were not considered when the techniques were first imagined and implemented for the adult brain. So far major developmental processes (e.g. axonal overproduction-pruning) remain unexplored, and complex situations (e.g. asynchronous myelination of crossing fibers) are insufficiently considered.

Regarding the development of white matter architecture, a first bottleneck relies on the accuracy of bundles reconstruction. As detailed previously, current tractography approaches are directly affected by the maturational stage. They provide limited information on fibers growth, pruning and crossings, and often mix these mechanisms at the voxel level. Yet, the known patterns of maturation might help to disentangle these complex situations: myelination progresses from neural soma to periphery, pruning and stabilized fibers have distinct myelination, fibers belonging to different bundles mature at different times, etc. Considering these maturational characteristics and asynchronies might facilitate the reconstruction of bundles and the differentiation between separate pools of fibers. A reverse bottleneck relies on the specificity of maturation measures within each bundle. Although promising, most univariate, multi-compartment or multi-parametric approaches provide aggregate measures at the voxel level, whereas a tract-level resolution should be achieved even in the complex places of bundles crossings.

Understanding the development of white matter networks might require studying both bundles architecture and maturation jointly, by modelling different pools of fibers with different spatial architectures, microstructural and myelination characteristics. Some aspects might be dealt with at the acquisition step. Recently, complex MR sequences have been implemented for the adult brain to explore fibers with different orientations based on diffusion MRI while measuring their myelin properties with $\mathrm{T} 1$ 
relaxometry [111]. Nevertheless, technological challenges remain to be addressed for infant imaging, for instance in terms of acquisition time, gradient and radiofrequency exposure. Alternatively, some implementations might be achieved in the post-processing steps, like constraining the quantification procedure by the bundles spatial trajectory (i.e. considering a certain degree of regularization of MRI parameters quantified along fibers).

\section{FUNCTIONAL MARKERS OF WHITE MATTER MYELINATION}

In parallel with the brain anatomical changes, important motor and cognitive development is observed [112], but so far, the links between functional progresses and the structural development of brain networks have been little investigated in typically developing children. A few recent studies have shown correlations between brain changes and psychomotor performances. For instance, cognitive scales for receptive and expressive language [113], and processing speeds [114] have been related to the white matter maturation quantified with MWF in fronto-temporal regions, and in the left occipital lobe respectively. The increasing accuracy and speed of processing complex sentences in children [115], and the performance in word learning in adults [116] have been related to the microstructural properties of the arcuate fasciculus connecting fronto-temporal regions of the language network.

But these behavioral measures which correspond to complex processes might only reflect the white matter maturation in an indirect way. Thus in this section, we focused on electrophysiological evoked responses for the visual, somatosensory and auditory modalities, with the hypothesis that age-related decreases in peak latencies (Fig. 5) are a good functional marker of white matter myelination (even if these changes also depend on the degree of cortical maturation and synaptogenesis). Actually, myelination is known to increase dramatically the conduction velocity of the nerve impulse along axonal fibers [56]. At constant pathway length, it leads to a decrease in the latency of brain responses throughout development. On the other hand, because brain size increases with age, mostly during the first two years, it may be necessary to further increase the conduction velocity by extending myelination just to maintain a correct latency [117].
Evoked responses (evoked potentials recorded with EEG or evoked fields recorded with MEG) correspond to the averaging over multiple trials. We have tried to homogenize the notation of peaks across studies to provide a coherent outline of age-related changes, since it sometimes corresponds either to the latency at which the peak occurs (e.g. P100 for a peak at $100 \mathrm{~ms}$ ), or to the peak number on the order of appearance after the stimulus (e.g. P1 for the first peak) which leads to a possible disparity between studies in infants at different ages and adults. Indeed, the morphology of evoked responses (i.e. the alternating series of positive and negative deflections) evolves dramatically throughout development and particularly during the preterm period, because of several mechanisms (microstructural organization of the cortical plate and subplate, development of intracortical connectivity, cortical folding, etc.). Important changes in the wave amplitudes are also observed in relation to synaptic bursting, pruning, etc. but also to exogenous factors such as skull calcification. Here, we considered how the gradual acceleration in the peak latencies during infancy might be used as a proxy of the bundles myelination. These studies are based on data obtained in the same subjects with $\mathrm{M} / \mathrm{EEG}$ on the functional side, and with diffusion imaging on the anatomical side. In some of these studies, both the bundles length and the response latency were considered to compute the conduction velocity of neural information.

\section{Visual system}

The visual modality develops mainly after birth, and infants' visual capacities drastically improve in a few months. Early visual cortical responses in newborns have been mostly studied using EEG recordings during pattern reversal stimulation. In adults, visual evoked potentials (VEPs) recorded in occipital regions at short latencies consist of a positive peak at around $100 \mathrm{~ms}$ (P100), surrounded by two negative peaks at around 70 and $145 \mathrm{~ms}$ (N70 and N145) [118]. At term birth, the small positive component P1 ( P100 in adults) is detected at a latency that decreases strongly and quickly with age [119-121], from around $260 \mathrm{~ms}$ in neonates to around $110-120 \mathrm{~ms}$ at $12-14$ weeks of age, depending on the patterns size [122] (Fig. 5a). The earlier negative component N1 $(\sim$ N70) develops in general after 2 weeks of age, and reaches a latency below $100 \mathrm{~ms}$ by 5 weeks of age [123, 124]. The second negative component $\mathrm{N} 2(\sim \mathrm{N} 145)$ is observed from 2 months of age 

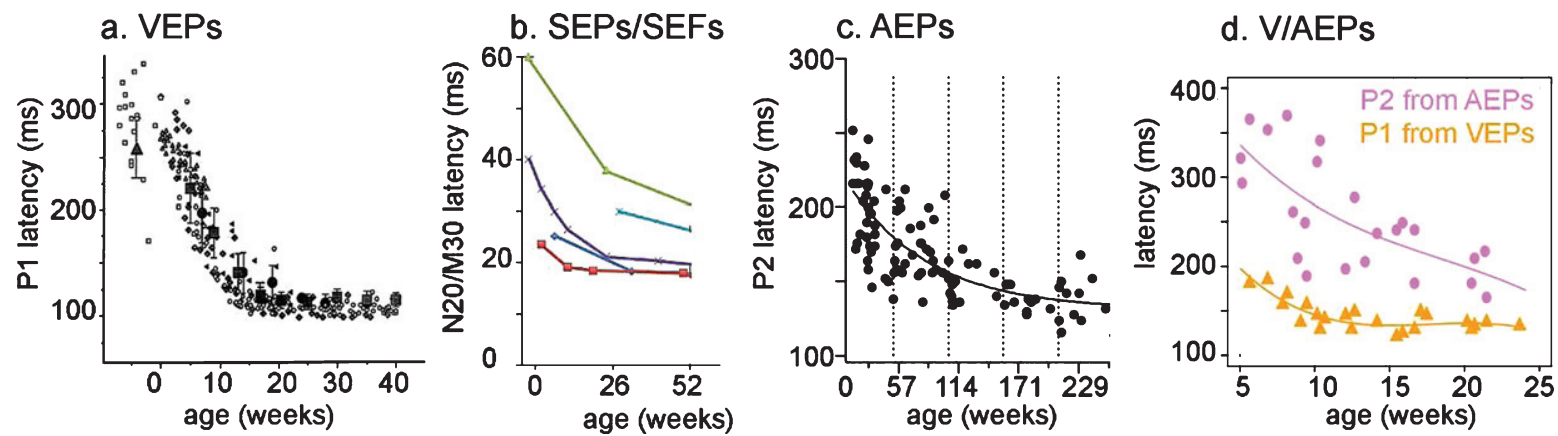

Fig. 5. Age-related changes in the latency of early evoked potentials. a: Latency of VEPs P1 during the first post-natal year, adapted from [122]. b: Latency of SEPs N20 and SEFs M30 during the first post-natal year, adapted from [139]. c: Latency of AEPs P2 during childhood, adapted from [140]. d: Latency comparison for P1 VEPs and P2 AEPs in infants.

[118]. P1 and N2 latencies reach their adult values around 7-12 months, whereas N1 latency decreases until 24-66 months [118].

These observations on VEPs responses suggest progressive increase in conduction velocity in afferent visual pathways related to fiber myelination [125], together with other maturational processes (e.g. retina and cortical development). A few recent studies have compared these functional changes to the microstructural evolution of visual bundles throughout development, focusing on the latency of P1 and its transfer from contralateral to ipsilateral hemisphere in case of lateral stimuli. During infancy, the increase in P1 conduction velocity has been related to the maturation of optic radiations (transverse diffusivity measured with DTI) [86]. This relation was specific (i.e. not observed for other white matter bundles) and not explained by intra-individual differences in infants' age. This study has been recently confirmed for visual lateral stimuli, and extended to corticocortical connections: the conduction velocity of P1 inter-hemispheric transfer was related to the maturation of the visual fibers of the corpus callosum [126]. In another study in 6-12-year-old children, the P1 latency from visual evoked fields (VEFs) was inversely related to the white matter integrity (FA measured with DTI) in visual and motor association regions [127]. Again this effect was independent of age differences across children, and it was not observed for N1 and N2 latencies.

Besides developmental studies, this issue of structure-function relationships has been also addressed in adults to characterize visual processing variability across subjects. A study combining VEFs and DTI has shown an inverse relationship between P1 latency and the microstructure (FA) of bilateral parietal and right lateral frontal white matter [128], so with a different spatial dependence than for children [127]. Based on VEPs and DTI, the inter-hemispheric transfer time of visual information for P1 (but not N1) has been inversely related to FA in the visual callosal fibers [129]. Nevertheless, these latter studies have not taken into account the inter-individual variations in the bundles length related to brain size and shape, which probably impact latency differences in a non-negligible way. Using diffusion imaging (AxCaliber approach), it has been proposed that the conduction velocity for $\mathrm{N} 2$ inter-hemispheric transfer was related to the axon diameter of visual callosal fibers [130]. Whereas such a demonstration in the human brain is very exciting, this study has been criticized in several aspects [131]: notably the estimated transfer times were very short $(\sim 5 \mathrm{~ms}), \mathrm{N} 2$ is a relatively late visual component, and earlier components would have been preferable in order to target activity in the primary visual cortex.

\section{Somatosensory and auditory systems}

So far, very few studies have related anatomical and functional maturation in the somatosensory and auditory modalities, despite intense changes in electrophysiological responses throughout infancy. Cortical somatosensory evoked potentials (SEPs) have been measured with EEG following electrical stimulation of the median nerve, at an intensity just above the motor threshold. In the adult brain, a negative peak (N20) and a positive peak (P25) are measured over centro-parietal electrodes at around 20 and $25 \mathrm{~ms}$ [132], and are supposed to reflect activity in the primary somatosensory cortex (S1) [133]. The SEPs development is intense in infants. At term birth, N20 is observed at around $25 \mathrm{~ms}$ [134], and its latency 
strongly decreases as a function of age during infancy $[134,135]$. P25 becomes the major component by 23 weeks of age [136]. More recently, somatosensory evoked magnetic fields (SEFs) have been measured with MEG. In adults, two early responses with opposite polarity are observed following median nerve or tactile stimulation (responses noted $\mathrm{N} 20 \mathrm{~m}$ and P30 m [137], or M30 and M50 [138]). In full-term newborns, two responses of the same polarity (M30 and M60) are detected with generators plausibly located in S1 [137] and latencies that decrease with age [139] (Fig. 5b). The maturation of SEPs/SEFs latencies have not been related to the myelination of corresponding white matter bundles so far. A single study in adults has related the inter-individual variability in SEPs-N140 inter-hemispheric transfer time to the axon diameter for callosal fibers supposed to connect $\mathrm{S} 1$ cortices [130], but these observations raised similar criticisms than for the visual system [131].

The auditory modality is already functional in utero, but its development is more protracted throughout infancy and toddlerhood than the visual and somatosensory modalities. In adults, auditory evoked potentials (AEPs) recorded at the vertex display several peaks: P1 ( 50-100 ms), N1 ( 100-150 ms), P2 ( 175-200 ms), N2 ( 270 ms), etc. [140, 141]. During the first months of life, the auditory response expands over almost all scalp electrodes and has large amplitude, making the comparison with adult components difficult. Two positive peaks are recorded over frontal electrodes, simultaneously to negative peaks over temporal and occipital electrodes at around $110-270 \mathrm{~ms}$ and $250-410 \mathrm{~ms}$ respectively. These latencies decrease exponentially with age [140, 142] (Fig. 5c). Regarding auditory evoked fields, a decrease in the latency of $\mathrm{P} 2 \mathrm{~m}$ is observed during infancy and toddlerhood [143]. This decrease has been related during childhood to the maturation of acoustic radiations based on DTI anisotropy [144]. Nevertheless, this correlation was not significant when taking into account the dependance of both measures on age. This casted doubt on the structurefunction specificity reported in this study.

\section{Perspectives}

Reliable correlations between the latency or conduction velocity of evoked responses and the myelination of underlying white matter bundles remain scarce so far. Further studies in the somatosensory and auditory modalities are required to confirm the results obtained in the visual modality. The comparison of anatomo-functional changes across modalities within the same subjects are needed to characterize the asynchronous development of brain networks and explore their sensitivity to distinct critical periods. For instance, the acceleration of early $\mathrm{M} / \mathrm{EEG}$ components seems more prolonged in the auditory modality than in the visual modality (Fig. 5d). It might be related to the different maturational tempos of the optic and acoustic radiations. If it is the case, M/EEG/DTI combination will provide in vivo assessments of the bundles functional integrity, a useful information in a lot of pathologies (premature births, autism, demyelinating diseases to cite a few). Aside from the latency of evoked responses, several other functional parameters might be compared to the brain anatomical changes throughout development. The evolution in morphology and amplitude of peaks, notably during the preterm period, might be related to microstructural changes in the cortex, and to the cortical expansion going with the folding process. Complexity measures of EEG signals (e.g. multiscale entropy which strongly increases throughout development [145]) might also be compared more robustly across functional modalities than evoked responses. While these measures in adults do not differ between the visual and auditory modalities, auditory responses show lower complexity than visual ones in children below 5 years of age [145]. Such discrepancies between networks need to be modelled to understand how integration is realized during development for example in the case of audio-visual integration during language acquisition. We advocate for a more systematic combination of DTI and M/EEG measures in the same children to explore these questions and open new understanding of human brain maturation and of its pathologies.

\section{CONCLUSION}

The early architecture and myelination of white matter networks have been detailed extensively with MRI in the recent years in fetuses, newborns and infants. Besides, the functional efficiency of neural communication has been assessed with M/EEG recordings for the sensory modalities, showing drastic declines in the response latencies throughout development. All these mechanisms occur at different times and speeds according to cerebral regions and involved functions. A few recent studies have aimed to relate the latency decrease, the increase in conduction velocity and the fibers myelination within 
a network, by combining investigations with complementary imaging techniques in the same children. Because the infants' age is the main factor to account for developmental changes at the brain and behavioral levels, multivariate analyses are required to highlight reliable anatomo-functional relationships. One should remind that these studies cannot decipher which is the cause or the consequence for these correlations. And many other factors may be responsible for inter-individual variability, like intra-uterine growth, gestational age at birth, socio-economic level and parental education, etc. Characterizing the individual differences across infants at the anatomical and functional levels is very challenging for understanding normal development, experience-dependent mechanisms and critical periods. But only systematic studies controlling these factors in healthy infants and children can provide robust biomarkers to detect early deviations in developmental trajectories related to perinatal perturbations or pathologies (e.g. premature birth, neonatal stroke), and follow the efficiency and robustness of medical interventions.

Multi-modal approaches might also provide insights on plasticity mechanisms occurring in the adult brain. Actually, even at the mature stage, transmission delays between different brain regions are dependent on the geometrical (i.e. length) and time computing (i.e. axonal diameter, myelination) properties of bundles, and thus on the areas of origin and target [146]. Since a few years, myelin-related mechanisms (formation, regulation and even remodeling) have appeared to contribute to circuit-level neural plasticity during adulthood, suggesting that unmyelinated and incompletely myelinated axons could be substrates for activity-dependent myelination according to environmental stimuli $[147,148]$. The resulting variations in conduction velocities might impact the functioning of neuronal networks not only in terms of the propagation of brain responses (e.g. spike-time arrival), but also of rhythmic activity (e.g. oscillation frequency, phase and amplitude, coupling and synchrony between oscillators) [149]. Thus accurate anatomo-functional models of networks would require to integer all these properties and account for spatial and temporal constraints [150].

\section{ACKNOWLEDGMENTS}

The authors' researches are supported by the "Fondation de France", the Fyssen Foundation, the "Fondation Motrice" and the McDonnell Foundation.

\section{CONFLICT OF INTEREST}

The authors declare no conflict of interest.

\section{REFERENCES}

[1] Smyser CD, Inder TE, Shimony JS, Hill JE, Degnan AJ, Snyder AZ, et al. Longitudinal analysis of neural network development in preterm infants. Cereb Cortex. 2010;20(12):2852-62.

[2] Doria V, Beckmann CF, Arichi T, Merchant N, Groppo M, Turkheimer FE, et al. Emergence of resting state networks in the preterm human brain. Proc Natl Acad Sci U S A. 2010;107(46):20015-20.

[3] Mahmoudzadeh M, Dehaene-Lambertz G, Fournier M, Kongolo G, Goudjil S, Dubois J, et al. Syllabic discrimination in premature human infants prior to complete formation of cortical layers. Proc Natl Acad Sci U S A. 2013;110(12):4846-51.

[4] Mahmoudzadeh M, Wallois F, Kongolo G, Goudjil S, Dehaene-Lambertz G. Functional Maps at the Onset of Auditory Inputs in Very Early Preterm Human Neonates. Cereb Cortex. 2016, in press.

[5] Sigaard RK, Kjaer M, Pakkenberg B. Development of the Cell Population in the Brain White Matter of Young Children. Cereb Cortex. 2016;26(1):89-95.

[6] Ng WP, Cartel N, Roder J, Roach A, Lozano A. Human central nervous system myelin inhibits neurite outgrowth. Brain Res. 1996;720(1-2):17-24.

[7] Dubois J, Dehaene-Lambertz G, Kulikova S, Poupon C, Hüppi PS, Hertz-Pannier L. The early development of brain white matter: A review of imaging studies in fetuses, newborns and infants. Neuroscience. 2014;276(C): 48-71.

[8] Dubois J, Kostovic I, Judas M. Development of structural and functional connectivity. In: Arthur W Toga, editor, Brain Mapping: An Encyclopedic Reference, Academic press: Elsevier. 2015;2:423-37.

[9] Vasung L, Huang H, Jovanov-Milosevic N, Pletikos M, Mori S, Kostovic I. Development of axonal pathways in the human fetal fronto-limbic brain: Histochemical characterization and diffusion tensor imaging. J Anat. 2010;217(4):400-17.

[10] Kostovic I, Jovanov-Milosevic N. The development of cerebral connections during the first 20-45 weeks' gestation. Semin Fetal Neonatal Med. 2006;11(6):415-22.

[11] Kostovic I, Rakic P. Developmental history of the transient subplate zone in the visual and somatosensory cortex of the macaque monkey and human brain. J Comp Neurol. 1990;297(3):441-70.

[12] Huang H, Zhang J, Wakana S, Zhang W, Ren T, Richards LJ, et al. White and gray matter development in human fetal, newborn and pediatric brains. Neuroimage. 2006;33(1):27-38.

[13] Judas M, Rados M, Jovanov-Milosevic N, Hrabac P, SternPadovan R, Kostovic I. Structural, immunocytochemical, and $\mathrm{mr}$ imaging properties of periventricular crossroads of growing cortical pathways in preterm infants. AJNR Am J Neuroradiol. 2005;26(10):2671-84.

[14] Huang H, Xue R, Zhang J, Ren T, Richards LJ, Yarowsky $\mathrm{P}$, et al. Anatomical characterization of human fetal brain development with diffusion tensor magnetic resonance imaging. J Neurosci. 2009;29(13):4263-73. 
[15] Xu G, Takahashi E, Folkerth RD, Haynes RL, Volpe JJ, Grant PE, et al. Radial Coherence of Diffusion Tractography in the Cerebral White Matter of the Human Fetus: Neuroanatomic Insights. Cereb Cortex. 2012.

[16] Miyazaki Y, Song JW, Takahashi E. Asymmetry of Radial and Symmetry of Tangential Neuronal Migration Pathways in Developing Human Fetal Brains. Front Neuroanat. 2016;10:2.

[17] Mitter C, Jakab A, Brugger PC, Ricken G, Gruber GM, Bettelheim D, et al. Validation of In utero Tractography of Human Fetal Commissural and Internal Capsule Fibers with Histological Structure Tensor Analysis. Front Neuroanat. 2015;9:164.

[18] Kostovic I, Judas M. Prolonged coexistence of transient and permanent circuitry elements in the developing cerebral cortex of fetuses and preterm infants. Dev Med Child Neurol. 2006;48(5):388-93.

[19] Vasung L, Lepage C, Rados M, Pletikos M, Goldman JS, Richiardi J, et al. Quantitative and Qualitative Analysis of Transient Fetal Compartments during Prenatal Human Brain Development. Front Neuroanat. 2016;10:11.

[20] Kostovic I, Petanjek Z, Judas M. Early areal differentiation of the human cerebral cortex: Entorhinal area. Hippocampus. 1993;3(4):447-58.

[21] Takahashi E, Folkerth RD, Galaburda AM, Grant PE. Emerging cerebral connectivity in the human fetal brain: An MR tractography study. Cereb Cortex. 2012;22(2):455-64.

[22] Kostovic I, Jovanov-Milosevic N, Rados M, Sedmak G, Benjak V, Kostovic-Srzentic M, et al. Perinatal and early postnatal reorganization of the subplate and related cellular compartments in the human cerebral wall as revealed by histological and MRI approaches. Brain Struct Funct. 2014;219(1):231-53.

[23] Schwartz ML, Goldman-Rakic PS. Prenatal specification of callosal connections in rhesus monkey. J Comp Neurol. 1991;307(1):144-62.

[24] Kostovic I, Kostovic-Srzentic M, Benjak V, JovanovMilosevic N, Rados M. Developmental dynamics of radial vulnerability in the cerebral compartments in preterm infants and neonates. Front Neurol. 2014; 5:139.

[25] Kostovic I, Judas M. The development of the subplate and thalamocortical connections in the human foetal brain. Acta Paediatr. 2010;99(8):1119-27.

[26] Rakic P, Bourgeois JP, Goldman-Rakic PS. Synaptic development of the cerebral cortex: Implications for learning, memory, and mental illness. Prog Brain Res. 1994;102:227-43.

[27] Innocenti GM, Price DJ. Exuberance in the development of cortical networks. Nat Rev Neurosci. 2005;6(12):955-65.

[28] LaMantia AS, Rakic P. Axon overproduction and elimination in the corpus callosum of the developing rhesus monkey. J Neurosci. 1990;10(7):2156-75.

[29] Petanjek Z, Judas M, Simic G, Rasin MR, Uylings HB, Rakic P, et al. Extraordinary neoteny of synaptic spines in the human prefrontal cortex. Proc Natl Acad Sci U S A. 2011;108(32):13281-6.

[30] Kasprian G, Brugger PC, Weber M, Krssak M, Krampl E, Herold C, et al. In utero tractography of fetal white matter development. Neuroimage. 2008;43(2):213-24.

[31] Pontabry J, Rousseau F, Oubel E, Studholme C, Koob M, Dietemann JL. Probabilistic tractography using Qball imaging and particle filtering: Application to adult and in-utero fetal brain studies. Med Image Anal. 2013;17(3):297-310.

[32] Bui T, Daire JL, Chalard F, Zaccaria I, Alberti C, Elmaleh $\mathrm{M}$, et al. Microstructural development of human brain assessed in utero by diffusion tensor imaging. Pediatr Radiol. 2006;36(11):1133-40.

[33] Mitter C, Prayer D, Brugger PC, Weber M, Kasprian G. In vivo tractography of fetal association fibers. PLoS One. 2015;10(3): 0119536.

[34] Partridge SC, Mukherjee P, Henry RG, Miller SP, Berman JI, Jin H, et al. Diffusion tensor imaging: Serial quantitation of white matter tract maturity in premature newborns. Neuroimage. 2004;22(3):1302-14.

[35] Dudink J, Lequin M, van Pul C, Buijs J, Conneman N, van Goudoever J, et al. Fractional anisotropy in white matter tracts of very-low-birth-weight infants. Pediatr Radiol. 2007;37(12):1216-23.

[36] van den Heuvel MP, Kersbergen KJ, de Reus MA, Keunen K, Kahn RS, Groenendaal F, et al. The Neonatal Connectome During Preterm Brain Development. Cereb Cortex. 2014.

[37] Ball G, Aljabar P, Zebari S, Tusor N, Arichi T, Merchant $\mathrm{N}$, et al. Rich-club organization of the newborn human brain. Proc Natl Acad Sci U S A. 2014;111(20):7456-61.

[38] Brown CJ, Miller SP, Booth BG, Andrews S, Chau V, Poskitt KJ, et al. Structural network analysis of brain development in young preterm neonates. Neuroimage. 2014;101:667-80.

[39] Markov NT, Ercsey-Ravasz M, Van Essen DC, Knoblauch K, Toroczkai Z, Kennedy H. Cortical high-density counterstream architectures. Science. 2013;342(6158):1238406.

[40] Kulikova S, Hertz-Pannier L, Dehaene-Lambertz G, Buzmakov A, Poupon C, Dubois J. Multi-parametric evaluation of the white matter maturation. Brain Struct Funct. 2015;220(6):3657-72.

[41] Dubois J, Hertz-Pannier L, Dehaene-Lambertz G, Cointepas Y, Le Bihan D. Assessment of the early organization and maturation of infants' cerebral white matter fiber bundles: A feasibility study using quantitative diffusion tensor imaging and tractography. Neuroimage. 2006;30(4): 1121-32.

[42] Geng X, Gouttard S, Sharma A, Gu H, Styner M, Lin W, et al. Quantitative tract-based white matter development from birth to age 2years. Neuroimage. 2012;61(3): 542-57.

[43] Dubois J, Poupon C, Thirion B, Simonnet H, Kulikova S, Leroy F, et al. Exploring the Early Organization and Maturation of Linguistic Pathways in the Human Infant Brain. Cereb Cortex. 2015.

[44] Yap PT, Fan Y, Chen Y, Gilmore JH, Lin W, Shen D. Development trends of white matter connectivity in the first years of life. PLoS One. 2011;6(9):e24678.

[45] Beaulieu C. The basis of anisotropic water diffusion in the nervous system - a technical review. NMR Biomed. 2002;15(7-8):435-55.

[46] Huppi PS, Maier SE, Peled S, Zientara GP, Barnes PD, Jolesz FA, et al. Microstructural development of human newborn cerebral white matter assessed in vivo by diffusion tensor magnetic resonance imaging. Pediatr Res. 1998;44(4):584-90.

[47] Wimberger DM, Roberts TP, Barkovich AJ, Prayer LM, Moseley ME, Kucharczyk J. Identification of premyelination by diffusion-weighted MRI. J Comput Assist Tomogr. 1995;19(1):28-33.

[48] Prayer D, Barkovich AJ, Kirschner DA, Prayer LM, Roberts TP, Kucharczyk J, et al. Visualization of non- 
structural changes in early white matter development on diffusion-weighted MR images: Evidence supporting premyelination anisotropy. AJNR Am J Neuroradiol. 2001;22(8):1572-6.

[49] Li G, Liu T, Ni D, Lin W, Gilmore JH, Shen D. Spatiotemporal patterns of cortical fiber density in developing infants, and their relationship with cortical thickness. Hum Brain Mapp. 2015.

[50] Brauer J, Anwander A, Perani D, Friederici AD. Dorsal and ventral pathways in language development. Brain Lang. 2013;127(2):289-95.

[51] Perani D, Saccuman MC, Scifo P, Anwander A, Spada D, Baldoli C, et al. Neural language networks at birth. Proc Natl Acad Sci U S A. 2011;108(38):16056-61.

[52] Zhang J, Evans A, Hermoye L, Lee SK, Wakana S, Zhang $\mathrm{W}$, et al. Evidence of slow maturation of the superior longitudinal fasciculus in early childhood by diffusion tensor imaging. Neuroimage. 2007;38(2):239-47.

[53] McCart RJ, Henry GH. Visual corticogeniculate projections in the cat. Brain Res. 1994;653(1-2):351-6.

[54] Van der Knaap MS, Valk J. Myelin and white matter. Magnetic resonance of myelin, myelination and myelin disorders, Van der Knaap MS and Valk J eds, SpringerVerlag, Berlin. 1995:1-17.

[55] Van der Knaap MS, Valk J. Myelination and retarded myelination. Magnetic resonance of myelin, myelination and myelin disorders, Van der Knaap MS and Valk J eds, Springer-Verlag, Berlin. 1995.

[56] Baumann N, Pham-Dinh D. Biology of oligodendrocyte and myelin in the mammalian central nervous system. Physiol Rev. 2001;81(2):871-927.

[57] Kinney HC, Brody BA, Kloman AS, Gilles FH. Sequence of central nervous system myelination in human infancy. II. Patterns of myelination in autopsied infants. J Neuropathol Exp Neurol. 1988;47(3):217-34.

[58] Flechsig P. Anatomie des Menschlichen Gehirn und Rückenmarks, auf myelogenetischer grundlage. G Thieme. 1920.

[59] Yakovlev PI, Lecours AR. The myelogenetic cycles of regional maturation in the brain. In: Minowski A eds Regional development of the brain in early life Blackwell, Oxford. 1967:3-69.

[60] Brody BA, Kinney HC, Kloman AS, Gilles FH. Sequence of central nervous system myelination in human infancy. I. An autopsy study of myelination. J Neuropathol Exp Neurol. 1987;46(3):283-301.

[61] Gilles F, Shankle W, Dooling E. Myelinated tracts: Growth patterns. Gilles F, Leviton A and Dooling E eds. 1983;John Wright PSG, Boston.

[62] Yakovlev PI. Morphological criteria of growth and maturation of the nervous system in man. Res Publ Assoc Res Nerv Ment Dis. 1962;39:3-46.

[63] Dubois J, Hertz-Pannier L, Cachia A, Mangin JF, Le Bihan D, Dehaene-Lambertz G. Structural asymmetries in the infant language and sensori-motor networks. Cereb Cortex. 2009;19(2):414-23.

[64] Mezer A, Yeatman JD, Stikov N, Kay KN, Cho NJ, Dougherty RF, et al. Quantifying the local tissue volume and composition in individual brains with magnetic resonance imaging. Nat Med. 2013;19(12):1667-72.

[65] Yeatman JD, Wandell BA, Mezer AA. Lifespan maturation and degeneration of human brain white matter. Nat Commun. 2014;5:4932.

[66] Matsumae M, Kurita D, Atsumi H, Haida M, Sato O, Tsugane R. Sequential changes in MR water proton relaxation time detect the process of rat brain myelination during maturation. Mech Ageing Dev. 2001;122(12): 1281-91.

[67] Barkovich AJ, Kjos BO, Jackson DE, Jr., Norman D. Normal maturation of the neonatal and infant brain: MR imaging at 1.5 T. Radiology. 1988;166(1 Pt 1):173-80.

[68] Kucharczyk W, Macdonald PM, Stanisz GJ, Henkelman RM. Relaxivity and magnetization transfer of white matter lipids at MR imaging: Importance of cerebrosides and $\mathrm{pH}$. Radiology. 1994;192(2):521-9.

[69] Haselgrove J, Moore J, Wang Z, Traipe E, Bilaniuk L. A method for fast multislice T1 measurement: Feasibility studies on phantoms, young children, and children with Canavan's disease. J Magn Reson Imaging. 2000;11(4):360-7.

[70] Engelbrecht V, Rassek M, Preiss S, Wald C, Modder U. Age-dependent changes in magnetization transfer contrast of white matter in the pediatric brain. AJNR Am J Neuroradiol. 1998;19(10):1923-9.

[71] Leppert IR, Almli CR, McKinstry RC, Mulkern RV, Pierpaoli C, Rivkin MJ, et al. T(2) relaxometry of normal pediatric brain development. J Magn Reson Imaging. 2009;29(2):258-67.

[72] Deoni SC, Dean DC, 3rd, O’Muircheartaigh J, Dirks $\mathrm{H}$, Jerskey BA. Investigating white matter development in infancy and early childhood using myelin water faction and relaxation time mapping. Neuroimage. 2012;63(3):1038-53.

[73] Poduslo SE, Jang Y. Myelin development in infant brain. Neurochem Res. 1984;9(11):1615-26.

[74] Huppi PS, Warfield S, Kikinis R, Barnes PD, Zientara GP, Jolesz FA, et al. Quantitative magnetic resonance imaging of brain development in premature and mature newborns. Ann Neurol. 1998;43(2):224-35.

[75] Huppi PS, Dubois J. Diffusion tensor imaging of brain development. Semin Fetal Neonatal Med. 2006;11(6): 489-97.

[76] Neil J, Miller J, Mukherjee P, Huppi PS. Diffusion tensor imaging of normal and injured developing human brain a technical review. NMR Biomed. 2002;15(7-8):543-52.

[77] Kersbergen KJ, Leemans A, Groenendaal F, van der Aa NE, Viergever MA, de Vries LS, et al. Microstructural brain development between 30 and 40 week corrected age in a longitudinal cohort of extremely preterm infants. Neuroimage. 2014;103:214-24.

[78] Nossin-Manor R, Card D, Raybaud C, Taylor MJ, Sled JG. Cerebral maturation in the early preterm period-A magnetization transfer and diffusion tensor imaging study using voxel-based analysis. Neuroimage. 2015;112:30-42.

[79] Dubois J, Dehaene-Lambertz G, Perrin M, Mangin JF, Cointepas Y, Duchesnay E, et al. Asynchrony of the early maturation of white matter bundles in healthy infants: Quantitative landmarks revealed noninvasively by diffusion tensor imaging. Hum Brain Mapp. 2008;29(1):14-27.

[80] Krogsrud SK, Fjell AM, Tamnes CK, Grydeland H, Mork $\mathrm{L}$, Due-Tonnessen $\mathrm{P}$, et al. Changes in white matter microstructure in the developing brain-A longitudinal diffusion tensor imaging study of children from 4 to 11 years of age. Neuroimage. 2015;124(Pt A):473-86.

[81] Nossin-Manor R, Card D, Morris D, Noormohamed S, Shroff MM, Whyte HE, et al. Quantitative MRI in the very preterm brain: Assessing tissue organization and myelination using magnetization transfer, diffusion tensor and T1 imaging. Neuroimage. 2013;64:505-16.

[82] Zanin E, Ranjeva JP, Confort-Gouny S, Guye M, Denis $\mathrm{D}$, Cozzone PJ, et al. White matter maturation of normal 
human fetal brain. An in vivo diffusion tensor tractography study. Brain Behav. 2011;1(2):95-108.

[83] Song SK, Sun SW, Ju WK, Lin SJ, Cross AH, Neufeld AH. Diffusion tensor imaging detects and differentiates axon and myelin degeneration in mouse optic nerve after retinal ischemia. Neuroimage. 2003;20(3):1714-22.

[84] Song SK, Sun SW, Ramsbottom MJ, Chang C, Russell J, Cross AH. Dysmyelination revealed through MRI as increased radial (but unchanged axial) diffusion of water. Neuroimage. 2002;17(3):1429-36.

[85] Song SK, Yoshino J, Le TQ, Lin SJ, Sun SW, Cross AH, et al. Demyelination increases radial diffusivity in corpus callosum of mouse brain. Neuroimage. 2005;26(1):13240.

[86] Dubois J, Dehaene-Lambertz G, Soares C, Cointepas Y, Le Bihan D, Hertz-Pannier L. Microstructural correlates of infant functional development: Example of the visual pathways. J Neurosci. 2008;28(8):1943-8.

[87] Johnson RT, Yeatman JD, Wandell BA, Buonocore MH, Amaral DG, Nordahl CW. Diffusion properties of major white matter tracts in young, typically developing children. Neuroimage. 2014;88C:143-54.

[88] Chen Z, Zhang H, Yushkevich PA, Liu M, Beaulieu C. Maturation Along White Matter Tracts in Human Brain Using a Diffusion Tensor Surface Model Tract-Specific Analysis. Front Neuroanat. 2016;10: Article no. 9.

[89] Berman JI, Mukherjee P, Partridge SC, Miller SP, Ferriero DM, Barkovich AJ, et al. Quantitative diffusion tensor MRI fiber tractography of sensorimotor white matter development in premature infants. Neuroimage. 2005;27(4):862-71.

[90] Li W, Wu B, Batrachenko A, Bancroft-Wu V, Morey RA, Shashi V, et al. Differential developmental trajectories of magnetic susceptibility in human brain gray and white matter over the lifespan. Hum Brain Mapp. 2014;35(6):2698-713

[91] McGowan JC. The physical basis of magnetization transfer imaging. Neurology. 1999;53(5 Suppl 3):S3-7.

[92] Xydis V, Astrakas L, Zikou A, Pantou K, Andronikou S, Argyropoulou MI. Magnetization transfer ratio in the brain of preterm subjects: Age-related changes during the first 2 years of life. Eur Radiol. 2006;16(1):215-20.

[93] Thiessen JD, Zhang Y, Zhang H, Wang L, Buist R, Del Bigio MR, et al. Quantitative MRI and ultrastructural examination of the cuprizone mouse model of demyelination. NMR Biomed. 2013;26(11):1562-81.

[94] Spader HS, Ellermeier A, O'Muircheartaigh J, Dean DC, 3rd, Dirks H, Boxerman JL, et al. Advances in myelin imaging with potential clinical application to pediatric imaging. Neurosurg Focus. 2013;34(4):E9.

[95] Melbourne A, Eaton-Rosen Z, Bainbridge A, Kendall GS, Cardoso MJ, Robertson NJ, et al. Measurement of myelin in the preterm brain: Multi-compartment diffusion imaging and multi-component T2 relaxometry. Med Image Comput Comput Assist Interv. 2013;16(Pt 2):336-44.

[96] Deoni SC, Matthews L, Kolind SH. One component? Two components? Three? The effect of including a nonexchanging free water component in multicomponent driven equilibrium single pulse observation of T1 and T2. Magn Reson Med. 2012;70(1):147-54.

[97] Deoni SC, Mercure E, Blasi A, Gasston D, Thomson A, Johnson M, et al. Mapping infant brain myelination with magnetic resonance imaging. J Neurosci. 2011;31(2): 784-91.

[98] Dean DC, 3rd, O'Muircheartaigh J, Dirks H, Waskiewicz N, Walker L, Doernberg E, et al. Characterizing longitu- dinal white matter development during early childhood. Brain Struct Funct. 2015;220(4):1921-33.

[99] Kulikova S, Hertz-Pannier L, Dehaene-Lambertz G, Poupon C, Dubois J. A new strategy for fast quantification of the fraction of water related to myelin: Application to infants imaging. PLoS One. 2016;11(10):e0163143.

[100] Assaf Y, Basser PJ. Composite hindered and restricted model of diffusion (CHARMED) MR imaging of the human brain. Neuroimage. 2005;27(1):48-58.

[101] Zhang H, Schneider T, Wheeler-Kingshott CA, Alexander DC. NODDI: Practical in vivo neurite orientation dispersion and density imaging of the human brain. Neuroimage. 2012;61(4):1000-16.

[102] Jelescu IO, Veraart J, Adisetiyo V, Milla S, Novikov DS, Fieremans E. One diffusion acquisition and different white matter models: How does microstructure change in human early development based on WMTI and NODDI? Neuroimage. 2015; 107:242-56.

[103] Kunz N, Zhang H, Vasung L, O'Brien KR, Assaf Y, Lazeyras F, et al. Assessing white matter microstructure of the newborn with multi-shell diffusion MRI and biophysical compartment models. Neuroimage. 2014;96: 288-99.

[104] Chang YS, Owen JP, Pojman NJ, Thieu T, Bukshpun P, Wakahiro ML, et al. White Matter Changes of Neurite Density and Fiber Orientation Dispersion during Human Brain Maturation. PLoS One. 2015;10(6):e0123656.

[105] Stikov N, Campbell JS, Stroh T, Lavelee M, Frey S, Novek $\mathrm{J}$, et al. In vivo histology of the myelin g-ratio with magnetic resonance imaging. Neuroimage. 2015;118:397-405.

[106] Melbourne A, Eaton-Rosen Z, Orasanu E, Price D, Bainbridge A, Cardoso MJ, et al. Longitudinal development in the preterm thalamus and posterior white matter: MRI correlations between diffusion weighted imaging and T2 relaxometry. Hum Brain Mapp. 2016;37(7):2479-92.

[107] Dean DC, 3rd, O'Muircheartaigh J, Dirks H, Travers BG, Adluru N, Alexander AL, et al. Mapping an index of the myelin g-ratio in infants using magnetic resonance imaging. Neuroimage. 2016;132:225-37.

[108] Prastawa M, Sadeghi N, Gilmore J, Lin W, Gerig G. A new framework for analyzing white matter maturation in early brain development. Proceedings of the 22nd IPMI meeting. 2010;97-100.

[109] Sadeghi N, Prastawa M, Fletcher PT, Wolff J, Gilmore $\mathrm{JH}$, Gerig G. Regional characterization of longitudinal DT-MRI to study white matter maturation of the early developing brain. Neuroimage. 2013;68:236-47.

[110] Lebenberg J, Poupon C, Thirion B, Leroy F, Mangin JF, Dehaene-Lambertz G, et al. Clustering the infant brain tissues based on microstructural properties and maturation assessment using multi-parametric MRI. IEEE ISBI. 2015; http://doi.org/10.1109/ISBI.2015.7163837:148-51.

[111] De Santis S, Barazany D, Jones DK, Assaf Y. Resolving relaxometry and diffusion properties within the same voxel in the presence of crossing fibres by combining inversion recovery and diffusion-weighted acquisitions. Magn Reson Med. 2016;75(1):372-80.

[112] Herschkowitz N. Brain development in the fetus, neonate and infant. Biol Neonate. 1988;54(1):1-19.

[113] O'Muircheartaigh J, Dean DC, 3rd, Ginestet CE, Walker L, Waskiewicz N, Lehman K, et al. White matter development and early cognition in babies and toddlers. Hum Brain Mapp. 2014;35(9):4475-87.

[114] Chevalier N, Kurth S, Doucette MR, Wiseheart M, Deoni SC, Dean DC, 3rd, et al. Myelination Is Associated 
with Processing Speed in Early Childhood: Preliminary Insights. PLoS One. 2015;10(10):e0139897.

[115] Skeide MA, Brauer J, Friederici AD. Brain Functional and Structural Predictors of Language Performance. Cereb Cortex. 2016;26(5):2127-39.

[116] Lopez-Barroso D, Catani M, Ripolles P, Dell'Acqua F, Rodriguez-Fornells A, de Diego-Balaguer R. Word learning is mediated by the left arcuate fasciculus. Proc Natl Acad Sci U S A. 2013;110(32):13168-73.

[117] Salami M, Itami C, Tsumoto T, Kimura F. Change of conduction velocity by regional myelination yields constant latency irrespective of distance between thalamus and cortex. Proc Natl Acad Sci U S A. 2003;100(10): 6174-9.

[118] Lippe S, Roy MS, Perchet C, Lassonde M. Electrophysiological markers of visuocortical development. Cereb Cortex. 2007;17(1):100-7.

[119] McCulloch DL, Skarf B. Development of the human visual system: Monocular and binocular pattern VEP latency. Invest Ophthalmol Vis Sci. 1991;32(8):2372-81.

[120] Harding GF, Grose J, Wilton A, Bissenden JG. The pattern reversal VEP in short-gestation infants. Electroencephalogr Clin Neurophysiol. 1989;74(1):76-80.

[121] Taylor MJ, Menzies R, MacMillan LJ, Whyte HE. VEPs in normal full-term and premature neonates: Longitudinal versus cross-sectional data. Electroencephalogr Clin Neurophysiol. 1987;68(1):20-7.

[122] McCulloch DL, Orbach H, Skarf B. Maturation of the pattern-reversal VEP in human infants: A theoretical framework. Vision Res. 1999;39(22):3673-80.

[123] Kraemer M, Abrahamsson M, Sjostrom A. The neonatal development of the light flash visual evoked potential. Doc Ophthalmol. 1999;99(1):21-39.

[124] Mercuri E, von Siebenthal K, Daniels H, Guzzetta F, Casaer P. Multimodality evoked responses in the neurological assessment of the newborn. Eur $\mathbf{J}$ Pediatr. 1994;153(9):622-31.

[125] Lee J, Birtles D, Wattam-Bell J, Atkinson J, Braddick O. Latency measures of pattern-reversal VEP in adults and infants: Different information from transient P1 response and steady-state phase. Invest Ophthalmol Vis Sci. 2012;53(3):1306-14.

[126] Adibpour P, Dehaene-Lambertz G, Dubois J. Relating the structural and functional maturation of visual and auditory white matter pathways with diffusion imaging and event-related potentials in infants. Proceedings of ISMRM. 2015:645.

[127] Dockstader C, Gaetz W, Rockel C, Mabbott DJ. White matter maturation in visual and motor areas predicts the latency of visual activation in children. Hum Brain Mapp. 2012;33(1):179-91.

[128] Stufflebeam SM, Witzel T, Mikulski S, Hamalainen MS, Temereanca S, Barton JJ, et al. A non-invasive method to relate the timing of neural activity to white matter microstructural integrity. Neuroimage. 2008;42(2): 710-6.

[129] Whitford TJ, Kubicki M, Ghorashi S, Schneiderman JS, Hawley KJ, McCarley RW, et al. Predicting interhemispheric transfer time from the diffusion properties of the corpus callosum in healthy individuals and schizophrenia patients: A combined ERP and DTI study. Neuroimage. 2011;54(3):2318-29.

[130] Horowitz A, Barazany D, Tavor I, Bernstein M, Yovel $\mathrm{G}$, Assaf Y. In vivo correlation between axon diameter and conduction velocity in the human brain. Brain Struct Funct. 2015;220(3):1777-88

[131] Innocenti GM, Caminiti R, Aboitiz F. Comments on the paper by Horowitz et al. (2014). Brain Struct Funct. 2015;220(3):1789-90.

[132] Mauguiere F, Allison T, Babiloni C, Buchner H, Eisen AA, Goodin DS, et al. Somatosensory evoked potentials. International Federation of Clinical Neurophysiology. 1999:79-90.

[133] Allison T, McCarthy G, Wood CC, Darcey TM, Spencer DD, Williamson PD. Human cortical potentials evoked by stimulation of the median nerve. I. Cytoarchitectonic areas generating short-latency activity. J Neurophysiol. 1989;62(3):694-710.

[134] Doria-Lamba L, Montaldi L, Grosso P, Veneselli E, Giribaldi G. Short latency evoked somatosensory potentials after stimulation of the median nerve in children: Normative data. J Clin Neurophysiol. 2009;26(3): 176-82.

[135] Laget P, Raimbault J, D'Allest AM, Flores-Guevara R, Mariani J, Thieriot-Prevost G. [Maturation of somesthetic evoked potentials in man]. Electroencephalogr Clin Neurophysiol. 1976;40(5):499-515.

[136] George SR, Taylor MJ. Somatosensory evoked potentials in neonates and infants: Developmental and normative data. Electroencephalogr Clin Neurophysiol. 1991;80(2):94-102.

[137] Lauronen L, Nevalainen P, Wikstrom H, Parkkonen L, Okada Y, Pihko E. Immaturity of somatosensory cortical processing in human newborns. Neuroimage. 2006;33(1):195-203.

[138] Pihko E, Nevalainen P, Stephen J, Okada Y, Lauronen L. Maturation of somatosensory cortical processing from birth to adulthood revealed by magnetoencephalography. Clin Neurophysiol. 2009;120(8):1552-61.

[139] Nevalainen P, Lauronen L, Pihko E. Development of Human Somatosensory Cortical Functions - What have We Learned from Magnetoencephalography: A Review. Front Hum Neurosci. 2015;8:158.

[140] Shafer VL, Yu YH, Wagner M. Maturation of cortical auditory evoked potentials (CAEPs) to speech recorded from frontocentral and temporal sites: Three months to eight years of age. Int J Psychophysiol. 2015;95(2): 77-93.

[141] Wunderlich JL, Cone-Wesson BK. Maturation of CAEP in infants and children: A review. Hear Res. 2006;212(12):212-23

[142] Chen A, Peter V, Burnham D. Auditory ERP response to successive stimuli in infancy. Peer J. 2016;4: e1580.

[143] Edgar JC, Murray R, Kuschner ES, Pratt K, Paulson DN, Dell $\mathrm{J}$, et al. The maturation of auditory responses in infants and young children: A cross-sectional study from 6 to 59 months. Front Neuroanat. 2015;9:131.

[144] Roberts TP, Khan SY, Blaskey L, Dell J, Levy SE, Zarnow DM, et al. Developmental correlation of diffusion anisotropy with auditory-evoked response. Neuroreport. 2009;20(18):1586-91.

[145] Lippe S, Kovacevic N, McIntosh AR. Differential maturation of brain signal complexity in the human auditory and visual system. Front Hum Neurosci. 2009;3: 48.

[146] Innocenti GM, Vercelli A, Caminiti R. The diameter of cortical axons depends both on the area of origin and target. Cereb Cortex. 2014;24(8):2178-88. 
[147] Chang KJ, Redmond SA, Chan JR. Remodeling myelination: Implications for mechanisms of neural plasticity. Nat Neurosci. 2016;19(2):190-7.

[148] Fields RD. A new mechanism of nervous system plasticity: Activity-dependent myelination. Nat Rev Neurosci. 2015;16(12):756-67.

[149] Pajevic S, Basser PJ, Fields RD. Role of myelin plasticity in oscillations and synchrony of neuronal activity. Neuroscience. 2014;276:135-47.
[150] Sanz-Leon P, Knock SA, Spiegler A, Jirsa VK. Mathematical framework for large-scale brain network modeling in The Virtual Brain. Neuroimage. 2015;111:385-430.

[151] Perrin M, Poupon C, Cointepas Y, Rieul B, Golestani N, Pallier C, et al. Fiber tracking in q-ball fields using regularized particle trajectories. Inf Process Med Imaging. 2005;19:52-63. 